\title{
TEORIA FISICA E SUA REALIFICAZIONE. CASO DI STUDIO: LA CAUSALITÀ
}

\author{
GIACOMO MAURO D’ARIANO (*)
}

\begin{abstract}
SunTO. - Si discute del realismo delle teorie classica e quantistica, rimarcando l'insostenibilità dell'ontologia dell'oggetto, e proponendone la sostituzione con la nozione di sistema delle teorie operazionali, nozione che rappresenta una connessione teorica fra due eventi. Nelle teorie operazionali la distinzione fra teoria e realtà obiettiva è ben delineata: la teoria fornisce la descrizione matematica dei sistemi e degli eventi, e di quest'ultimi predice la probabilità congiunta; la realtà oggettiva si identifica con la collezione degli eventi effettivamente accaduti. Si analizzano quindi alcuni casi di realificazione della teoria - ovvero la fallacia dell'identificazione della teoria con la realtà. In particolare si approfondisce il caso della nozione di causalità e della relativa relazione causale fra eventi, rimarcandone la natura prettamente teorica, contrariamente alla diffusa attribuzione di oggettività. Si ristabilisce il ruolo della causalità in fisica, in quanto teorema della teoria quantistica, e quindi anche della teoria classica (che della teoria quantistica è restrizione), mostrando che essa rappresenta la generalizzazione probabilistica del concetto comunemente utilizzato nella teoria della relatività ristretta, e discutendo come tale nozione possa trivializzarsi nella teoria classica. Si conclude con una critica della Past Hypothesis di David Albert riguardo la natura del tempo, e della relativa visione a Block Universe dello spazio-tempo, per riaffermare la Realtà del Tempo.
\end{abstract}

$$
* * *
$$

ABSTRACT. - I will discuss realism of classical and quantum theories, assessing the untenability of the object ontology, and proposing its substitution with the notion of system used in operational theories, notion that represents a theoretical connection between two events. Within operational theories the distinction between theory and objective reality is well defined: the theory provides the mathematical description of systems and events, and predicts the joint probability of the events; objective reality is identified with the collection of events that actually occurred. I then analyse some cases of realification of the theory - namely the fallacy of identifying theory with reality. In particular, the cases of the notion of causality and causal connection between events are

(*) Istituto Lombardo Accademia di Scienze e Lettere, Milano, Dipartimento di Fisica, Università degli Studi di Pavia, Italia. E-mail: dariano@unipv.it 
analysed, emphasising their purely theoretical nature, contrarily to the widespread connotation of objectivity. I re-establish the role of causality in physics as a theorem of quantum theory, and hence also of classical theory (which is a restriction of quantum theory), showing how it represents a probabilistic generalisation of the same concept used in special relativity, and discussing why such notion may trivialise in the classical case. I end with a critique of David Albert's Past Hypothesis about the nature of time, and of the resulting Block Universe vision of space-time, to reaffirm Reality of Time.

\section{PREMESSA TERMINOLOGICA}

Nel seguito utilizzerò il termine realtà con la connotazione implicita di verificabilità ed oggettività. Più precisamente, con il termine realtà oggettiva ${ }^{1}$ intenderò una realtà condivisibile, verificabile unanimemente ad esempio l'esito testa o croce del lancio di una moneta, o il numero rappresentato da un frequenzimetro digitale. Di contro, solitamente con il termine realtà si intende ciò che esiste effettivamente, in contrapposizione a ciò che è illusorio, immaginario o fittizio - ad esempio contrapposto al sogno o al credo. Per denotare la cosa in se', che generalmente non può essere percepita in modo oggettivo ma può essere concepita intellettivamente, useremo quando necessita il termine noumeno, il quale tipicamente corrisponde ad un elemento teorico. Ad esempio: l'onda elettronica o elettromagnetica, la particella, l'elettrone, sono tutti da considerarsi noumeni, non realtà oggettive, quali ad esempio sono invece le scintillazioni su un tubo catodico, o le tracce visibili in una camera a bolle.

\section{LE LEZIONI METODOLOGICHE DELLA TEORIA QUANTISTICA}

\section{Distinzione fra esperimento e teoria: crisi del realismo}

La lezione metodologica più profonda che deriva dalla teoria quantistica è la necessità logica di tenere ben distinti esperimento e teo-

1 Si noti che fra obiettivo e oggettivo esiste una lieve differenza di significato. Obiettivo è aggettivo che indica imparzialità. Oggettivo indica che sta alla realtà dei fatti, concreto, che si fonda su dati sperimentabili, che si attiene ai fatti senza intervento del soggetto. Ma poiché in questo contesto useremo il termine realtà intendendola come indipendente dal soggetto, i due termini diventano effettivamente sinonimi, e verranno usati interscambiabilmente. 
ria, ovvero realtà obiettiva condivisibile da un lato, e teoria con la quale la descriviamo e prevediamo, dall'altro. Tale distinzione è cruciale per l'applicabilità del criterio di demarcazione di Popper basato sulla falsificabilità della teoria [Pop59]. Prima della teoria quantistica la distinzione fra realtà e teoria non costituiva un problema, in quanto nella fisica classica ogni elemento della teoria ha un corrispondente nella realtà oggettiva. Per questo classifichiamo quella classica come teoria realistica. La teoria quantistica ha messo in crisi questa forma di realismo, in quanto non si concilia con l'esistenza della non località, la quale sancisce l'impossibilità di interpretare l'osservazione come lettura di una realtà locale pre-esistente l'osservazione. ${ }^{2}$ La lezione della non località è che il forzare l'osservazione ad essere la lettura di una realtà oggettiva preesistente l'osservazione stessa, richiede che tale presunta realtà debba dipendere da operazioni eseguite remotamente - operazioni generalmente non conoscibili, anche in linea di principio: una vittoria di Pirro per il realismo. A peggiorare la situazione vi è il fatto che tale dipendenza da operazioni remote è istantanea, e quindi, in accordo alla relatività ristretta, l'operazione remota avviene prima o dopo l'osservazione, a seconda della scelta del sistema di riferimento. ${ }^{3}$

\section{La probabilità quantistica non è mancanza di conoscenza}

Non è quindi possibile interpretare la probabilità nella misurazione come mancanza di conoscenza di una realtà locale oggettiva pre-esistente la misurazione stessa, interpretazione sintetizzata dall'espressione inglese lack of knowledge. Il risultato della misurazione, non potendo essere la lettura passiva di una realtà oggettiva locale, è pertanto generato all'atto stesso della misurazione. Ciò non significa che il risultato non dipenda da qualcosa di pre-esistente la misurazione, altrimenti ne sarebbe vanificata la finalità. Il risultato della misurazione dipende infatti dallo stato quantistico del sistema sul quale la misurazione è eseguita. Possiamo quindi vedere lo stato quantistico come un noumeno non oggettivabile preesi-

2 Rammento che per locale si intende indipendente da operazioni eseguite remotamente.

3 Non sussiste quindi alcuna connotazione causale della dipendenza da operazioni remote, come sembrerebbe implicare l'espressione Einsteniana: spooky action at distance. 
stente la misurazione. Esso rappresenta quello che chiamiamo anche informazione quantistica, ovvero informazione privata conosciuta solo da chi ha preparato lo stato quantistico: informazione non clonabile (si veda il teorema del no cloning quantistico [DY96]), informazione quantistica crittografica. Informazione quantistica privata, posseduta prima della misurazione da chi ha preparato lo stato, in contrasto con l'informazione classica pubblica, condivisibile, acquisita con l'osservazione.

Probabilismo inerente: attuazione condivisibile di potenzialità

Si è detto che l'osservazione non si può considerare come mera lettura di una realtà locale oggettiva preesistente: il risultato della misurazione è generato all'atto dell'osservazione, come attualizzazione oggettiva di una potenzialità soggettiva. L'informazione quantistica privata collassa all'atto dell'osservazione in informazione classica pubblica, ovvero si trasforma nell'informazione classica condivisibile corrispondente al risultato della misura. Una tale attualizzazione condivisibile di una potenzialità non condivisa è il mistero del collasso della funzione d'onda del postulato di von Neumann [vN96], che tormenta la comunità dei fisici teorici sin da quando Schrödinger introdusse il suo famoso paradosso del gatto. $\mathrm{Ma}$ è anche l'unica possibile traduzione del probabilismo inerente della teoria quantistica, probabilismo non interpretabile come lack of knowledge, quindi realtà oggettiva generata all'atto della misurazione. Si potrebbe pensare che il determinismo, implicando corrispondenza esatta delle diverse realtà a tempi differenti, rappresenti l'Essere Parmenideo, mentre il probabilismo inerente appare come l'unico modo di superare il determinismo e rappresenta quindi il Divenire Eracliteo.

\section{Olismo quantistico}

L'entanglement quantistico conduce al cosiddetto olismo della teoria. L'olismo della teoria quantistica si presenta in due forme equivalenti nelle due pictures (di Heisenberg per le osservabili, e di Schrödinger per gli stati).

Nella Heisenberg picture, ovvero in termini di osservabili si ha:

Esistono proprietà del tutto che sono incompatibili con ogni proprietà delle parti. 
Nella teoria quantistica una proprietà è associata ad un proiettore ortogonale su un sottospazio di Hilbert. Un proiettore entangled congiunto di due parti non commuta con nessun proiettore di ogni singola parte, ovvero è incompatibile con essi nel senso di Heisenberg's (come posizione e momento di una particella). In aggiunta, le menzionate proprietà del tutto rappresentano il caso "tipico" all'interno della teoria, nel senso che il set degli effetti congiunti per cui non si ha olismo è di misura nulla nel set di tutti gli effetti [Loc02]. ${ }^{4}$

Nella Schrödinger picture l'olismo si traduce nell'affermazione:

\section{La conoscenza del tutto non implica la conoscenza delle parti.}

Ciò consegue dall'esistenza di stati congiunti di oggetti composti per i quali l'informazione estraibile è maggiore della somma delle informazioni che si possono estrarre da ogni oggetto componente separatamente. Nella teoria quantistica l'informazione estraibile da un set di oggetti visti come un tutt'uno è fornita dal loro stato quantistico congiunto, mentre l'informazione estraibile da ogni oggetto separatamente è data dal suo stato quantistico marginale. Anche in questo caso la situazione olistica è tipica, esattamente come per la Heisenberg picture. La situazione estrema è quella di uno stato massimamente entangled puro di due oggetti, i cui stati marginali sono massimamente misti: lo stato puro corrisponde a conoscenza massima (ad esempio conoscenza del valore esatto di un set massimale di osservabili commutanti), mentre lo stato massimamente misto corrisponde a conoscenza nulla (ad esempio distribuzioni uniformi dei valori delle osservabili).

Esistono poi situazioni paradossali, quale ad esempio la violazione del pigeonhole principle [ACP+16], laddove esistono stati di tre particelle che possono stare solo in due scatole, stati per i quali la probabilità di trovare due particelle nella stessa scatola è nulla!

La lezione che deriva dall'olismo quantistico è che l'ontologia dell'oggetto non è sostenibile, in quanto la nozione di oggetto identificato come collezione di proprietà non è compatibile con la connotazione mereologica di componibilità degli oggetti a formare oggetti composti. La familiare nozione di oggetto diviene uno sconveniente epiciclo

4 Per il concetto di effetto si veda la sezione IV. 
Tolemaico, e la rivoluzione Copernicana consiste nel sostituire la nozione di oggetto con la nozione di sistema, come vedremo nel seguito.

\section{REALIFICAZIONE DELLA TEORIA}

Il termine soggettivo produce sovente reazioni negative di non scientificità nei fisici, e, in generale, in chi si occupa di scienza. Non stupisce quindi che generalmente la teoria fisica venga implicitamente considerata con una connotazione di obiettività. Strettamente parlando, invece:

La teoria è un'estensione del processo di inferenza logica. Essa permette di fare previsioni sulla base di conoscenze di dati di fatto, nonché di ipotesi.

E, innegabilmente, le ipotesi non necessariamente sono condivise o condivisibili. La teoria stessa è un ipotesi, la quale potrebbe essere falsificata, e in tale caso, perderebbe la sua condivisibilità. L'obiettività, che rappresenta il trademark della scientificità stessa, oltre che valere per le regole di inferenza logica, è un requisito necessario per il dato sperimentale, ma non può riguardare la condivisione delle ipotesi, inclusa la teoria stessa. E proprio la richiesta di oggettivazione delle ipotesi spesso rappresenta la motivazione della realificazione della teoriatecnicamente ipostatizzazione della teoria: ovvero "la fallacia della concretezza fuori luogo", ovvero il concretismo del trattare la teoria stessa come reale ed oggettiva. Ma se la teoria fosse tale, non potrebbe essere falsificabile.

\section{Realificazione delle probabilità}

Il concetto stesso di probabilità rappresenta l'esempio canonico di realificazione di elementi teorici, allorquando si parla di interpretazioni della nozione di probabilità [Gil00], contrapponendo la "interpretazione" Bayesiana soggettivista con quella frequentista oggettivista, quest'ultima presupponendo una probabilità oggettiva che può essere "misurata". $\mathrm{Al}$ contrario, il valore della probabilità di un evento è sempre

5 Questo è anche il caso dell'interpretazione di propensità di Popper, dove la 
determinato dall'informazione a priori in possesso di chi ne stima il valore sulla base della sua conoscenza di dati di fatto e delle ipotesi da lui assunte. Tipicamente, nello stimare una probabilità ci si riconduce alla regola di equiprobabilità di Laplace, ovvero all'equivalenza di un set completo di possibili eventi: in altri termini, ci si basa su un'ipotesi di simmetria. Anche quando si stima il valore di una probabilità mediante prove ripetute, il valore stimato è conseguenza dell'ipotesi di interscambiabilità delle prove, e come dimostrato da Bruno de Finetti, tale ipotesi corrisponde ad assumere a priori una miscela di valori per la probabilità che viene stimata. Infatti, se si considera solo la logica e l'oggettività del risultato della prova ripetuta, senza ulteriore ipotesi, si può al più affermare che se un evento è obiettivamente accaduto la sua probabilità è strettamente maggiore di zero, mentre se non è accaduto la sua probabilità è strettamente minore di uno. Ma non è possibile affermare logicamente bounds più stringenti dei valori 0 e 1 , e men che meno è possibile affermare che la probabilità possiede un preciso valore obiettivo. Come è sempre stato, sin dalle origini della sua nozione basata sul gioco d'azzardo, la probabilità è semplicemente una regola normativa di decision-making basata su ipotesi e sulla conoscenza di dati di fatto. La probabilità è teoria.

\section{Realismo e realificazione del determinismo}

La richiesta di realismo della teoria - ovvero la corrispondenza degli elementi della teoria con elementi della realtà oggettiva - è essa stessa una realificazione della teoria. E tale richiesta induce la realificazione del determinismo, ovvero la richiesta che la realtà sia deterministica, e le probabilità si interpretino come lack of knowledge, e il probabilismo non sia inerente.

\section{IL CASO DELLA CAUSALITÀ IN FISICA}

La realificazione del determinismo e l'identificazione dei concetti di determinismo e causalità ha condotto ad abolire la nozione stessa di

probabilità è supposta connessa ad un meccanismo obiettivo, laddove invece il meccanismo è teoria, ed in quanto tale non oggettiva. 
causalità, la quale rappresenta un ingrediente fondamentale per tutta la scienza, e che viene utilizzata nel quotidiano bagaglio di regole di inferenza. Riporto quello che sostiene il mainstream divulgativo di fisica teorica, in un popolare libro di Sean Carrol [Car16]:

Abbiamo già evidenziato come la legge di conservazione di Laplace 6 mina il ruolo centrale posto da Aristotele sulla causalità.

È la visione completamente deterministica di Laplace. E Carrol così continua:

Concetti come causa non appaiono in nessun caso nelle equazioni di Newton, ne' nella nostra moderna formulazione delle leggi della Natura. Ma non possiamo negare che l'idea che un evento sia causato da un altro è molto naturale ed apparentemente si adatta bene al modo in cui viviamo il mondo. Questa apparente discrepanza può essere fatta risalire all'entropia e alla freccia del tempo.

Della freccia del tempo parlerò nel seguito. Carrol prosegue quindi con la popolare citazione di Bertrand Russel, che viene reiterata nella letteratura contraria alla causalità ${ }^{7}$

La legge della causalità, credo, come le molte cose che passano ai filosofi, è una reliquia dell'età del passato, sopravvive come la monarchia, perché è erroneamente supposta non fare alcun danno. [Rus12]

Come vedremo nel seguito, contrariamente a quanto afferma Russel, la causalità è un teorema della teoria quantistica, ed è sperimentalmente falsificabile. Inoltre, una corretta nozione di causalità deve essere generalmente probabilistica, contrariamente alla menzionata fallace identificazione con il concetto di determinismo, e deve supportare la connotazione Einsteniana di possibilità di “comunicazione" fra eventi distinti.

6 Qui Carrol intende la reversibilità dell'evoluzione dinamica.

7 Qui Carrol fa leva sul cosiddetto effetto Matthew, ovvero, acquisire credito citando uno scienziato molto stimato quale il grande logico-matematico Bertrand Russel. 


\section{LA FORMULAZIONE DELLA TEORIA QUANTISTICA COME TEORIA OPERAZIONALE}

La formulazione più conveniente della teoria quantistica è quella di teoria operazionale. La teoria operazionale è un'estensione della teoria delle probabilità, che alle probabilità congiunte di eventi multipli associa anche una connettività fra essi. Le connessioni dall'output di un evento all'input di un altro evento sono i cosiddetti sistemi della teoria. In accordo alla teoria delle probabilità, ogni evento deve essere considerato come elemento di una collezione completa di possibili eventi alternativi, detta test. Quale evento sia effettivamente avvenuto (l'outcome) rappresenta l'elemento osservabile della teoria. La teoria operazionale deve provvedere la descrizione matematica degli eventi e dei sistemi, e mediante tale descrizione associare al circuito la probabilità congiunta di tutti i possibili outcomes. Tale probabilità costituisce la predizione della teoria.

La nozione di sistema corrisponde quindi ad una connessione teorica input-output fra due eventi diversi. Il sistema viene quindi rappresentato da un link che connette due eventi, direzionato dall'output del primo evento verso l'input del secondo. Il grafo complessivo che ne risulta è unidirezionato aciclico. ${ }^{8}$ Inoltre, il grafo è chiuso, poichè ogni sistema connette eventi. ${ }^{9}$ La teoria è quindi formulata in termini di un grafo aciclico unidirezionato (DAG: directed acyclic graph) di connessioni fra eventi, quello che normalmente è chiamato circuito quantistico. Per ottenere un circuito chiuso si utilizzano particolari eventi che non hanno input, detti preparazioni, ed eventi che non hanno output, detti osservazioni.

Ma cosa si intende precisamente quando affermiamo che gli eventi sono connessi da sistemi? Si intende che la probabilità congiunta degli eventi dipende generalmente da come essi sono connessi, e conseguentemente anche le distribuzioni di probabilità marginali di un singolo test o di un gruppo di tests ${ }^{10}$ generalmente dipendono dalla rete di con-

8 Poiché le composizioni sequenziali e in parallelo di eventi corrispondono ad un nuovo evento, l'esistenza di cicli darebbe luogo a coppie di eventi connessi in modo contraddittorio sia dall'input dell'uno all'output dell'altro, che viceversa.

9 Consideriamo qui solo grafi finiti.

10 Rammentiamo che la distribuzione di probabilità marginale di un gruppo di tests si ottiene sommando le probabilità congiunte sui possibili risultati degli altri tests. 
nessioni completa, che contiene anche i tests marginalizzati. Torneremo su questo in seguito.

Nel linguaggio operazionale gli stati sono classi di equivalenza probabilistiche ${ }^{11}$ di eventi di preparazione, mentre le classi di equivalenza di osservazioni si chiamano effetti, e le classi di equivalenza di eventi generici sono genericamente dette trasformazioni.

In quanto teoria operazionale la teoria quantistica è un estensione della teoria delle probabilità, la quale a sua volta è un estensione della logica. ${ }^{12}$ Non si tratta quindi, come pensava von Neumann con il suo programma di quantum logic condiviso con Birkoff, di modificare la logica, bensì di estenderla. La teoria quantistica è quindi l'ampliamento di un processo di inferenza.

Una teoria operazionale è anche una teoria dell'informazione. Ciò può essere compreso pensando al circuito come il run di un programma di calcolo, laddove i tests sono le subroutines ed i sistemi sono i registri di memoria mediante i quali le subroutines si scambiano dati, ovvero rappresentano le connessioni input-output fra le subroutines. Seguendo questa linea, il lavoro [CDP11] deriva la teoria quantistica da sei principi "informatici". ${ }^{13}$ Uno dei sei postulati "informatici" da cui discende la teoria è il postulato di causalità che qui analizziamo. In precedenti memorie presentate in questa stessa Accademia si sono analizzati i sei postulati, e se ne è discussa la rilevanza epistemologica, notando come tutti i sei postulati riguardano la possibilità di falsificare proposizioni della teoria con operazioni locali. I sei postulati informatici della teoria si possono inversamente derivare come teoremi dalla formulazione assiomatica di von Neumann. Pertanto, la causalità è un teorema della teoria quantistica nella formulazione di von Neumann.

11 Due eventi sono probabilisticamente equivalenti se avvengono con la stessa probabilità congiunta all'interno dello stesso circuito.

12 La teoria della probabilità può essere vista come un'estensione delle regole di inferenza della logica. Richard T. Cox[Cox61] ha dedotto le regole dell'inferenza probabilistica a partire dall'algebra Booleana delle proposizioni, incluse regole di somma, prodotto, e regola di Bayes. Si veda anche il testo postumo di Edwin Jaynes[Jay03].

13 Il lavoro [CDP11], oggetto di approfondite analisi da parte della comunità internazionale, è divenuto un libro di testo sulla teoria quantistica. Il testo è stato presentato presso questa stessa Accademia dal Prof. Attilio Rigamonti il 6 aprile 2017. 


\section{La causalità, principio falsificabile della teoria quantistica}

Consideriamo il semplice circuito quantistico in Fig. 1 costituito da due apparati di Stern-Gerlach in cascata preceduti dalla preparazione di un fascio di particelle a spin $1 / 2$ con orientazione casuale. I due apparati di Stern-Gerlach disposti in cascata all'output del generatore del fascio di particelle sono entrambi test binari, corrispondenti a coppie di risultati opposti per il verso dello spin. Ognuno dei tests è un ben preciso apparato sperimentale, e l'evento per ognuno dei due SternGerlach è identificato con l'apparato in questione all'occorrenza dello specifico risultato.

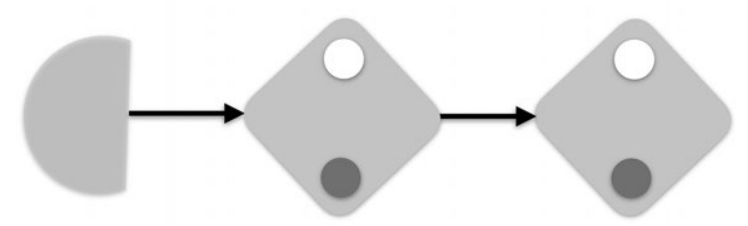

Fig. 1. Circuito quantistico costituito da due apparati di Stern-Gerlach (quadrati) in cascata preceduti dalla preparazione di un fascio di particelle a spin $1 / 2$ con orientazione casuale (semicerchio). Sulla base alla teoria quantistica immaginiamo la particella con spin $1 \frac{1}{2}$ transitare attraverso $i$ due apparati Stern-Gerlach, producendo l'accensione del led corrispondente al rivelatore di particelle presso uno dei due pinholes attraverso il quale sarebbe transitata la particella. Rappresentiamo il percorso della particella (o onda) tracciando una linea direzionata che unisce $i$ due box che rappresentano gli apparati. Un tale segmento direzionato è quello che chiamiamo sistema.

Sulla base alla teoria quantistica immaginiamo la particella con spin $1 / 2$ transitare attraverso i due apparati Stern-Gerlach, producendo l'accensione del led corrispondente al rivelatore di particelle presso uno dei due pinholes attraverso il quale sarebbe transitata la particella. In realtà, come avrebbe rimarcato Niels Bohr, noi non vediamo transitare ne' particelle, ne' onde: osserviamo solo l'accensione del led. La particella è nella nostra immaginazione: è un elemento della teoria. Rappresentiamo il percorso della particella (o onda) tracciando una linea direzionata che unisce i due box che rappresentano gli apparati. Un tale segmento direzionato è quello che chiamiamo sistema. In tal senso il sistema e una nozione prettamente teorica, e rappresenta una connessione fra due eventi distinti.

Vediamo ora cosa si intende per causalità della teoria, con un esperimento concepito per falsificarla. 
Consideriamo un setup sperimentale (che chiameremo esperimento $A$ ), che consiste in un generatore di fascio di particelle, tutte con spin up, seguito da due apparati di Stern-Gerlach in cascata, entrambi con il gradiente di campo magnetico verticale. E consideriamo un secondo setup (che chiameremo esperimento B) nel quale il secondo Stern-Gerlach della cascata ha il gradiente orizzontale, anziché verticale (Fig. 2). Il generatore di fascio di particelle seguito dal primo Stern-Gerlach rappresenta una nuova preparazione di particelle, mentre il secondo Stern-Gerlach in cascata è una osservazione del fascio. La teoria soddisfa il principio di causalità se [DCP17]:

Causalità: la probabilità della preparazione è indipendente dalla scelta dell'osservazione.
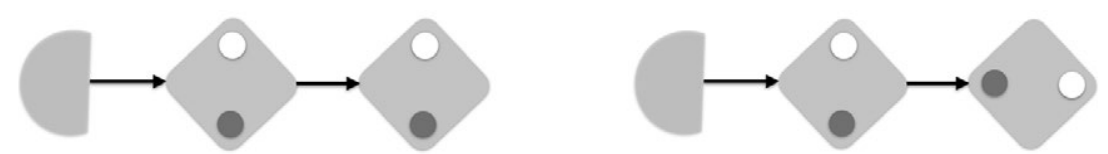

Fig. 2. Due diversi esperimenti: esperimento $A$ (a sinistra) ed esperimento B (a destra). Si veda il testo.

Nel nostro caso ciò vorrebbe dire che la distribuzione di probabilità del primo Stern-Gerlach della cascata non dipende da quale SternGerlach lo segue, sia esso con gradiente verticale o orizzontale.

Nell'esperimento in questione, quindi, la causalità sarebbe falsificata se, ad esempio, il primo evento della cascata è sempre up nell'esperimento $\mathrm{A}$, ma si può verificare un evento down nell'esperimento $\mathrm{B}$, ovvero la distribuzione di probabilità del primo Stern-Gerlach dipende dall'orientazione del gradiente del secondo Stern-Gerlach. La teoria quantistica ci garantisce che ciò non può avvenire (la dimostrazione generale del teorema di causalità è riportata in appendice, tratta dalla referenza originale [D'A18]).

Ne concludiamo che la causalità è falsificabile: è quindi scienza, non pseudoscenza, come implicherebbe Bertrand Russel nell'articolo [Rus12].

Riassumiamo brevemente l'apparato concettuale che supporta la nozione di causalità. Abbiamo detto che la particella è solo nella nostra immaginazione: è un elemento teorico. Abbiamo tracciato una linea 
direzionata che unisce due apparati di Stern Gerlach: tale segmento direzionato è quello che chiamiamo sistema. Il sistema quindi è una nozione prettamente teorica, che rappresenta una connessione fra due eventi distinti. Normalmente la nozione di sistema viene considerata alla stessa stregua di quella di oggetto, ma è invero una nozione molto più ampia. E come abbiamo appreso dalla lezione dell'olismo della teoria quantistica la nozione di oggetto all'interno della teoria quantistica non è sostenibile. Inoltre la connotazione della nozione di oggetto è, come dice la parola stessa, "oggettiva", mentre quella di sistema è teorica. Ma la formulazione operazionale della teoria quantistica risolve la crisi del realismo con una separazione ben definita degli elementi teorici (sistemi ed eventi) da quelli sperimentali (quali eventi sono effettivamente accaduti). Non c'è più corrispondenza fra di gli elementi della teoria con elementi della realtà obiettiva condivisibile.

L'ingrediente non banale da rimarcare riguardo il concetto di causalità è che in una teoria operazionale la dipendenza parametrica dal circuito della probabilità congiunta degli eventi implica che anche le probabilità marginali su tests del circuito generalmente dipendono ancora dal test marginalizzato. Ora, siccome ogni preparazione seguita da trasformazioni è ancora una preparazione (e similmente un'osservazione preceduta da trasformazioni è ancora un'osservazione), ogni circuito chiuso si può riguardare come la composizione di una preparazione seguita da un'osservazione (Fig. 3). Quello che afferma il postulato di causalità è che la probabilità marginale della preparazione è indipendente dalla scelta del test di osservazione (mentre il viceversa, ovviamente, non è generalmente vero).

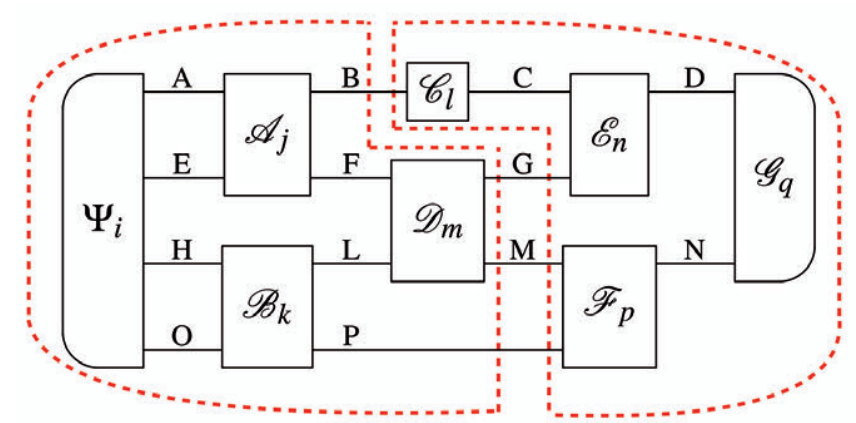

Fig. 3. Ogni circuito chiuso è equivalente alla composizione di una preparazione (porzione tratteggiata sulla sinistra) con una osservazione (porzione tratteggiata sulla destra). 


\section{Esempi di errori storici dovuti all'assenza del postulato di causalità}

Il postulato di causalità può apparire ovvio, ma non lo è per nulla, ed è molto potente. Come vedremo subito, vieta le comunicazioni a distanza senza interazione. Si deve qui ricordare che nel 1982 Nick Herbert [Her82] proponeva sulla rivista Foundation of Physics un metodo di comunicazione istantanea a distanza basato sulla condivisione di uno stato entangled. ${ }^{14}$ Il metodo era basato sulla possibilità di: a) condividere un singoletto di stati di polarizzazione di fotone, b) eseguire misurazioni locali di polarizzazione, c) clonare gli stati di polarizzazione mediante amplificazione stimolata di radiazione. L'emissione stimolata produce davvero copie perfette di fotoni polarizzati: l'errore di Nick Herbert fu però di sottovalutare l'effetto dell'emissione spontanea. Purtroppo, la verifica teorica dell'impossibilità di clonare fotoni polarizzati utilizzando emissione stimolata è alquanto complessa, e il risultato fu che l'articolo, esaminato da una dozzina di referees, venne pubblicato anche se errato. Alcuni mesi dopo apparve sulla rivista Nature una breve comunicazione di Wootters e Zurek [WZ82] che dimostrava che il cloning perfetto di stati era impossibile perché contraddiceva la linearità della teoria quantistica. Questo no-cloning theorem, comunque, pur mostrando che l'analisi di Nick Herbert era scorretta, non dimostrava nulla circa l'impossibilità di comunicare secondo il suo schema, in quanto il cloning perfetto è possibile probabilisticamente, o, deterministicamente è possibile in modo imperfetto, ma gli errori di comunicazione possono essere corretti mediante codifica se la probabilità di errore per bit non è uguale ad $1 / 2$. Quindi, il no cloning theorem non dimostrava comunque l'impossibilità di comunicare superluminalmente secondo lo schema proposto da Nick Herbert.

Occorre qui ricordare che il fraintendimento dell'entanglement con la possibilità di comunicare istantaneamente era errore comune nella comunità dei fisici all'epoca del lavoro di Herbert, ed in parte era indotto anche dall'espressione spiritosa di Einstein Spooky action at distance (azione "fantasmatica" a distanza). E la non ovvietà all'epoca dell'impossibilità di comunicare istantaneamente in presenza della non-località quantistica è dimostrata dall'espressione di Abner Shimony: peaceful coexistence between quantum mechanics and special relativity [Shi83, Shi86].

14 Herbert brevettò tale metodo ottenendo anche finanziamenti dal Pentagono, come riportato sul libro di David Kaiser How the Hippies Saved Physics[Kai11]. 
L'impossibilità di comunicare senza interazione condividendo uno stato entangled è invece semplicemente una conseguenza immediata della causalità. Il circuito che descrive la condivisione di uno stato entangled fra Alice e Bob che eseguono osservazioni/trasformazioni locali può essere diviso in una preparazione che include lo stato entangled e anche la misura di Alice, e in una osservazione, che contiene solo la misurazione di Bob, e l'indipendenza della probabilità di preparazione di Alice dalla scelta della osservazione di Bob sancisce l'impossibilità per Bob di comunicare ad Alice. Lo stesso ragionamento si può fare scambiando simmetricamente i ruoli di Alice e Bob. Ne concludiamo che è impossibile comunicare solo condividendo uno stato: occorre avere interazione (Figg. 4 e 5).

Come ricordato precedentemente, tutti e sei i postulati "informatici" della teoria quantistica hanno una rilevanza epistemologica, e riguardano la falsificabilità delle proposizioni della teoria in situazione di controllo locale. La causalità stessa è necessaria per garantire un cono spaziotemporale di schermatura dell'esperimento, garantendo che la falsificazione non sia dovuta ad un'azione a distanza o dal futuro.

Notiamo infine che la presente nozione di causalità corrisponde anche al suo uso standard nei processi di inferenza e modellizzazione (si veda il libro di Judea Pearl [Pea12]).
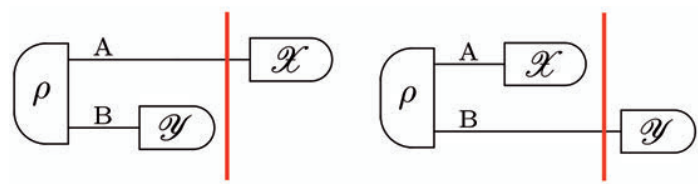

Fig. 4. Dimostrazione grafica dell'impossibilità di comunicare istantaneamente a distanza mediante la condivisione di uno stato entangled. Nel diagramma a sinistra, la preparazione $\rho$ (corrispondente allo stato entangled) insieme all'osservazione YY di Bob costituiscono una nuova preparazione, la cui probabilità congiunta è quindi indipendente (per la causalità), dalla osservazione X di Alice. Pertanto quel che Bob rivela è indipendente dalla operazione di Alice, e Alice non comunica nulla a Bob. La situazione è la stessa nel diagramma a destra, con i ruoli di Alice e Bob scambiati.

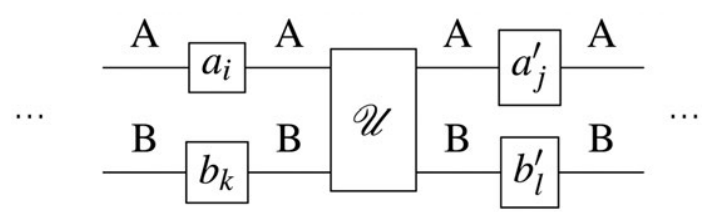

Fig. 5. Schema di possibile comunicazione fra alice e Bob, che coinvolge un interazione (denotata dal simbolo Ul in figura). 


\section{Causalità quantistica e relativistica}

Il principio di causalità della teoria quantistica è equivalente al concetto Einsteniano utilizzato in relatività ristretta. Infatti, il principio di causalità può essere riformulato come segue:

\section{Diciamo che l'evento $\mathcal{A}$ precede l'evento $\mathcal{F}$ quando $\mathcal{A}$ è connesso a $\mathcal{F}$ da un percorso unidirezionato da un output di $\mathcal{A}$ a un input di $\mathcal{F}$. Diremo equivalentemente che l'evento $\mathcal{F}$ segue l'evento $\mathcal{A}$.}

La relazione d'ordine segue è una relazione d'ordine parziale fra eventi, e in quanto tale definisce un cono. Nel nostro caso si tratta del cono causale di output, ovvero l'insieme di tutti gli eventi che lo seguono l'evento assegnato. Simmetricamente la relazione precede definisce il cono causale di input di un evento.(si veda Fig. 6) Si noti che relazione d'ordine parziale e il cono sono in corrispondenza biunivoca, nel senso che la relazione d'ordine parziale definisce il cono, e simmetricamente il cono assegna l'ordinamento parziale: ad esempio possiamo affermare che l'evento $\mathcal{F}$ segue l'evento $\mathcal{A}$ se e solo se appartiene al suo cono di output.

Se ora identifichiamo la freccia input-output con quella del tempo, ${ }^{15}$ il cono di input equivale al cono passato di un evento, e quello di output al cono futuro. Possiamo ora riformulare il principio di causalità nel modo seguente:

Una teoria operazionale è causale se e solo se la probabilità marginale di ogni evento è indipendente da quali eventi possono accadere all' infuori del suo cono passato.

Quest'ultima formulazione della causalità è la versione probabilistica della nozione di causalità usata in relatività ristretta, laddove si prevede che un evento spazio-temporale possa essere causalmente (e quindi temporalmente) preceduto da un altro solo se si trova nel suo cono futuro.

Si noti che, come nella teoria della relatività ristretta, anche per la teoria operazionale si possono definire foliazioni sul circuito, anziché nello spazio tempo. Se aggiungiamo le ulteriori richieste di località, omogeneità, ed isotropia alla rete causale di eventi (la località consiste

15 Una tale freccia del tempo si accorda con la nozione di memoria, poiché la memoria di un evento è connessa all'output dell'evento stesso. 
nel requisito che ogni evento sia connesso ad un insieme finito di eventi), allora dalla rete causale ritroviamo esattamente la forma dei coni causali della relatività ristretta [DT13]. Ma per ritrovare le trasformazioni di Lorentz dobbiamo anche richiedere che la rete causale sia un circuito quantistico, ovvero sia quello che chiamiamo un automa quantistico cellulare. E qui menziono solamente che aggiungendo ulteriori principi che riguardano il circuito quantistico, quali omogeneità, località, isotropia, e linearità nel campo, si ottiene la teoria di campo libera (si veda la mia memoria del 2016 presentata presso questa Accademia nella giornata di studio su Realtà senza Realismo [D'A17]).
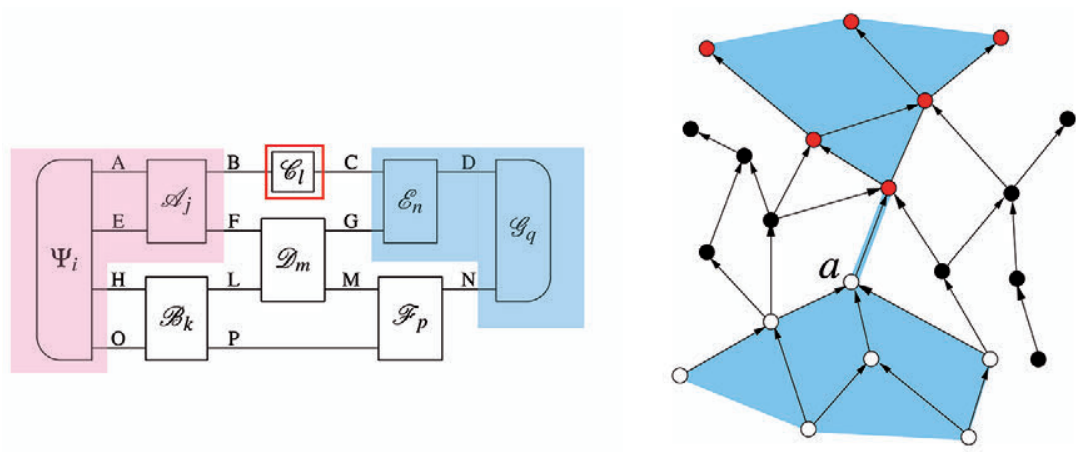

Fig. 6. Coni causali di un evento. Sulla sinistra i coni passato (rosa) e futuro (azzurro) dell'evento $\mathrm{Cl}$ nel circuito in Fig. 3. Sulla destra i coni passato e futuro dell'evento a nella rappresentazione usuale di rete causale generica (da Ref.[DT13]).

\section{La causalità è un principio anche della teoria classica}

Abbiamo visto la formulazione del principio di causalità come indipendenza della probabilità marginale dei test di preparazione dalla scelta dei tests di osservazione, o, brevemente: indipendenza delle preparazioni dalle osservazioni. Abbiamo argomentato che una tale affermazione distilla tutte le forme in cui la causalità appare in fisica. Abbiamo visto che il principio di causalità è un teorema della teoria quantistica. Qui rimarchiamo che il teorema vale anche per la teoria classica, in quanto restrizione della teoria quantistica (si veda l'Appendice). ${ }^{16}$

16 Qui si intende la teoria classica nella sua formulazione operazionale, ovvero senza la connotazione "meccanica". Tale teoria è equivalente alla teoria dell'informazione 
Perchè, allora, sembra che non esista il principio di causalità, a quanto afferma Carroll? Nel caso classico il principio di causalità è trivializzato dal realismo della teoria (corrispondenza biunivoca fra elementi della teoria ed elementi della realtà). Inoltre, il restringersi allo scenario deterministico mette cause ed effetti (preparazioni ed osservazioni) in corrispondenza biunivoca, trivializzandone la distinzione. D'altra parte, la teoria classica rende la nozione di "misurazione" irrilevante, identificandola con la semplice "lettura" dello stato, che viene quindi identificato con il risultato della misurazione. Ne consegue che è possibile una sola scelta di osservazione, e la formulazione in termini di indipendenza della preparazione dalla scelta dell'osservazione si trivializza nell'esistenza di un'unica osservazione possibile, mentre in teoria quantistica la presenza di complementarità introduce la possibilità di osservazioni diverse.

Cosa significa allora "causalità" nel caso classico? Benchè nel mondo classico si ha un solo tipo di osservazione, è comunque possibile scegliere la preparazione, e ciò rende appropriata la formulazione del principio di causalità come indipendenza di un evento dagli eventi fuori dal suo cono passato. E, in effetti, abbiamo visto che una tale formulazione è condivisa dalla relatività speciale, e il principio di causalità corrisponde all'usuale formulazione Einsteniana. In base alla stessa formulazione la causalità esclude i potenziali anticipati dell'elettromagnetismo.

Concludendo, ricordiamo che la causalità è una nozione genuinamente teorica, che discende da una teorizzazione delle connessioni fra gli eventi, le quali sono quindi stabilite da un ulteriore substrato teorico, quale appunto la teoria di Maxwell. Lo spazio tempo Minkowskiano di per se non supporta una struttura causale definita, ma è la teoria soggiacente (in questo caso quella di Maxwell), che stabilisce quali eventi possono essere connessi causalmente, e quali no. Ovviamente, l'estensione dell'argomento al caso della gravità necessiterebbe una formulazione alla Laplace con propagazione del campo gravitazionale. La formulazione Einsteniana della relatività generale non si accorda invece alla formulazione operazionale, in quanto presenta soluzioni cicliche, quali quelle di Gödel.

classica. Il principio di causalità si estende comunque alla meccanica classica (incluso elettromagnetismo): per questo si veda anche la nota a pie' pagina n. 18 . 
La causalità determina la freccia del tempo

La causalità stabilisce la freccia del tempo, identificando il cono di input come il cono passato, e quello di output come cono futuro. Anche l'irreversibilità stessa del processo di misurazione (ovvero il collasso della funzione d'onda) corrisponde ad una connessione causale fra eventi: la preparazione dello stato seguita irreversibilmente dall'evento osservato.

Ma per chi non crede nella causalità (e quindi non crede nella teoria quantistica, oppure non l'ha capita), la freccia del tempo si ottiene in un altro modo. È questo il caso della Past Hypothesis di David Albert. Sempre dallo stesso libro di Sean Carrol [Car16] leggiamo:

La ragione per cui c'è una netta differenza fra sopra e sotto per noi non è per la natura stessa dello spazio, ma perché viviamo in prossimità di un oggetto molto influente: la Terra. Il tempo funziona alla stessa maniera. Nel mondo di tutti i giorni la freccia del tempo è ben chiara, ed è comprensibile che tu creda che esista una differenza intrinseca fra passato e futuro. In realtà, entrambe le direzioni del tempo sono create uguali. La ragione per la quale sussiste una differenza cosi evidente fra passato e futuro non è nella natura del tempo, ma perché viviamo nella ripercussione di un evento estremamente influente: il Big Bang.

Quel che dobbiamo aggiungere è una assunzione circa le condizioni iniziali dell'universo osservabile, ovvero che esso originariamente era in uno stato di entropia molto bassa. Il filosofo David Albert ha soprannominato questa assunzione "Past Hypothesis". Quel che sappiamo è che questo stato iniziale di entropia bassa è responsabile per la freccia termodinamica del tempo.

Peccato che la Past Hypothesis non sia in alcun modo falsificabile. La visione che ne deriva è quella cosiddetta Block Universe sostenuta da alcuni relativisti, fra i quali Carlo Rovelli [Rov17], Julian Barbour [Bar00], e altri ancora: tutto il tempo è equivalente al presente. Paul Davies scrive [Dav12]:

Obiettivamente, ${ }^{17}$ passato presente e futuro devono essere uguali. Tutta l'eternità è disposta in un blocco uni-dimensionale composto di tempo e tree dimensioni spaziali.

17 Sottolineo la parola "obiettivamente": ecco la realificazione della teoria! 
Ma per lo stesso ragionamento di David Albert potremmo allora dire che se dopo il Big Bang ci fosse un Big Crunch (perché, ad esempio, l'universo è chiuso), allora come oggi vediamo un uovo frantumarsi cadendo al suolo, e parimenti vediamo un onda quantistica collassare nel risultato di una misurazione, in futuro potremmo vedere l'uovo ricomporsi, e così vedere tutti gli eventi scomparire e ritornare nel noumeno dell'onda.

\section{APPENDICE - DimOSTRAZIONE DEL TEOREMA DI CAUSALITÀ DELLA TEORIA QUANTISTICA}

Una formulazione completa di una teoria operazionale è data fornendo la descrizione matematica dei sistemi e delle trasformazioni della teoria. In tale framework l'assiomatizzazione di von Neumann della teoria quantistica ${ }^{18}$ può essere formulata come segue: ${ }^{19}$

Teoria Quantistica: ogni sistema A è associato a uno spazio di Hilbert $\mathcal{H}_{\mathrm{A}}$, e la composizione di sistemi è rappresentata dal prodotto tensore dei rispettivi spazi (da cui segue che per il sistema triviale I si ha $\mathcal{H}_{\mathrm{I}}=\mathbb{C}$ ). Una transformazione $\mathcal{T} \in \operatorname{Transf}(\mathrm{A} \rightarrow \mathrm{B})$ è descritta da una mappa completamente positiva $(\mathrm{CP})$ trace-non-increasing da $\mathrm{T}\left(\mathcal{H}_{\mathrm{A}}\right)$ a $\mathrm{T}\left(\mathcal{H}_{\mathrm{B}}\right)$, e la trasformazione è deterministica quando la mappa è trace-preserving.

18 Si noti che per Teoria Quantistica qui si intende la teoria dei sistemi astratti, senza l'originale connotazione "meccanica" della teoria, nel qual caso essa è usualmente chiamata Meccanica Quantistica. Il passaggio dalla teoria astratta dei sistemi alla meccanica avviene attraverso la teoria di campo, trattando il campo quantistico come collezione numerabile di sistemi in interazione. Questo è il procedimento seguito in Refs. [DP14, BDP16], dove si ricava la teoria di campo libera (Weyl, Dirac, Maxwell), aggiungendo richieste di semplicità riguardo la topologia del circuito (località, omogeneità, isotropia), nonché la linearità delle trasformazioni in funzione del campo. La teoria di campo ottenuta equivale alla versione discreta della teoria algebrica di campo [Haa96], che nel contesto operazionale corrisponde all'algebra complessa degli effetti, con la teoria classica corrispondente ad algebra Abeliana.

19 Utilizziamo la seguente comune notazione: $\operatorname{Bnd}(\mathcal{H})\left(\operatorname{Bnd}^{+}(\mathcal{H})\right)$ denota l'insieme degli operatori limitati sullo spazio di Hilbert $\mathcal{H} ; \mathrm{T}(\mathcal{H})\left(\mathrm{T}^{+}(\mathcal{H})\right)$ denota l'insieme degli operatori (positivi) di classe traccia su $\mathcal{H} ; \mathrm{T}_{\leq 1}^{+}(\mathcal{H})$ denota il set degli operatori positivi su $\mathcal{H}$ con traccia minore o uguale a $1 ; \operatorname{Transf}(\mathrm{A} \rightarrow \mathrm{B})$ è il set delle trasformazioni dal sistema $\mathrm{A}$ al sistema $\mathrm{B}, \mathrm{St}(\mathrm{A})$ è il set degli stati del sistema $\mathrm{A}, \mathrm{Eff}(\mathrm{A})$ è il set degli effetti.Tutte le mappe nel seguito sono lineari. 
Gli stati e gli effetti sono casi speciali di trasformazioni, con sistema triviale di input i primi, e di output i secondi. Abbiamo quindi che:

1. Il set degli stati del sistema $A$ è $\operatorname{St}(A):=\operatorname{Transf}(\mathrm{I} \rightarrow \mathrm{A})$. Ne consegue che gli stati di A sono rappresentati da mappe positive $e^{20}$ da $[0,1]$ a $\mathrm{T}_{\leq 1}^{+}\left(\mathcal{H}_{\mathrm{A}}\right)$, e gli stati deterministici corrispondono a traccia unitaria. In particolare $\mathrm{St}(\mathrm{I}) \equiv \mathrm{T}_{\leq 1}^{+}(\mathbb{C}) \equiv[0,1]$ sono valori di probabilità.

2. Il set degli effetti del sistema $A$ è $\operatorname{Eff}(\mathrm{A})=\operatorname{Transf}(\mathrm{A} \rightarrow \mathrm{I})$. Segue che gli effetti di A sono mappe positive da $\mathrm{T}_{\leq 1}^{+}\left(\mathcal{H}_{\mathrm{A}}\right)$ a $[0,1]$, quindi sono funzionali della forma $\varepsilon_{i}(\cdot)=\operatorname{Tr}_{\mathrm{A}}[\cdot E]$, dove $\operatorname{Tr}_{\mathrm{A}}$ denota la traccia parziale su $\mathcal{H}_{\mathrm{A}}$ e $\mathrm{Bnd}^{+}\left(\mathcal{H}_{\mathrm{A}}\right) \ni E \leq I_{\mathrm{A}}$. $\operatorname{Tr}_{\mathrm{A}}$ è l'unico effetto deterministico di A.

Quando detto è tutto ciò che occorre sapere della teoria quantistica. Dobbiamo ora rimarcare che la teoria classica può anch'essa essere formulata come teoria operazionale [DCP17], ed è la restrizione della teoria quantistica ai simplessi massimali di stati contenuti nel convesso degli stati quantistici, e restringendo le trasformazioni in accordo. ${ }^{21}$ Segue che la teoria classica può essere formulata come segue:

Teoria Classica: È la teoria operazionale restrizione della teoria quantistica che corrisponde a scegliere $\operatorname{Transf}(\mathrm{A} \rightarrow \mathrm{B})=\mathrm{T}\left(n_{\mathrm{A}}, n_{\mathrm{B}}\right)$ il set delle matrici $n_{\mathrm{A}} \times n_{\mathrm{B}}$ sub-stocastiche di Markov, $\operatorname{con} n_{\mathrm{A}}=\operatorname{dim}\left(\mathcal{H}_{\mathrm{A}}\right)$.

\section{Il teorema di causalità}

Riportiamo la formulazione del principio di causalità in una teoria operazionale [DCP17]:

Causalità: la probabilità della preparazione è indipendente dalla scelta dell'osservazione.

20 Per sistema triviale di input o di output la mappa è CP se preserva la positività.

21 La teoria classica riformulata come teoria operazionale è la restrizione della teoria quantistica dove il set degli stati $\mathrm{St}(\mathrm{A})$ è limitato al $\operatorname{simplesso} \mathrm{S} \operatorname{dim}\left(\mathcal{H}_{A}\right)$ dato dal convex hull di un set prefissato massimale di stati quantistici puri ortogonali, e restringendo le transformazioni in accordo. 
Dimostriamo ora che il principio di causalità è soddisfatto dalla teoria quantistica.

Theorem IV.1 La teoria quantistica soddisfa il principio di causalità.

\section{Dimostrazione}

Essendo $\operatorname{Tr}_{\mathrm{A}}$ l'unico effetto deterministico per A, si ha $\sum_{j \in \mathrm{Y}} E_{j}=I_{\mathrm{A}}$ per qualunque test di osservazione. La probabilità congiunta di preparazione e di osservazione è data da

$$
p(i, j):=\operatorname{Tr}\left[E_{j} \rho_{i}\right]
$$

e la probabilità marginale della preparazione è

$$
\sum_{j \in Y} p(i, j)=\sum_{j \in Y} \operatorname{Tr}\left[\rho_{i} E_{j}\right]=\operatorname{Tr}\left[\rho_{i}\right]
$$

che è indipendente dalla scelta dell'osservazione $\left\{E_{j}\right\}_{j \in \mathrm{Y}}$ Q $Q E D$

Si noti l'asimmetria fra preparazione e osservazione. Infatti, diversamente dall'Eq. (2), la probabilità marginale della osservazione è data da

$$
\sum_{j \in \mathrm{x}} p(i, j)=\sum_{j \in \mathrm{x}} \operatorname{Tr}\left[\rho_{i} E_{j}\right]=\operatorname{Tr}\left[\rho_{\mathrm{x}} E_{j}\right]
$$

che generalmente dipende dalla preparazione $\left\{\rho_{i}\right\}_{j \in \mathrm{x}}$ attraverso $\rho_{\mathrm{x}}=\sum_{j \in \mathrm{x}} \rho_{i}$.

È immediato verificare che per una teoria operazionale qualunque si ha la seguente forma equivalente del principio di causalità:

Principio di causalità (forma equivalente): Una teoria operazionale soddisfa il principio di causalità se e solo se l'effetto deterministico è unico, e dipende solo dal sistema della teoria.

Infatti, come visto nella dimostrazione del teorema IV.1, valutare la probabilità marginale della preparazione corrisponde a calcolare il coarse-graining del test di osservazione, il quale equivale all'effetto deterministico. Pertanto, l'indipendenza dell'effetto deterministico dal test di osservazione equivale all'indipendenza della probabilità marginale della preparazione dalla scelta dell'osservazione. 
Nel testo [DCP17] si mostra anche come il principio di causalità è equivalente alla possibilità di "normalizzare" gli stati, il che, a sua volta, è equivalente alla possinilità di preparare ogni stato deterministicamente, ad esempio mediante post-selezione..$^{22}$ Infine, si mostra come il postulato di causalità restringe la struttura convessa della teoria, con il convesso degli stati in forma di cono troncato da un iperpiano di codimensione 1, e il convesso degli effetti a forma di "fuso" intersezione fra il cono degli effetti e in cono che corrisponde all'essere dominati dell'effetto deterministico.

\section{RINGRAZIAMENTI}

Questo lavoro è stato possibile con il supporto della John Templeton Foundation (Grant ID No. 43796: A Quantum-Digital Universe, and Grant ID No. 60609: Quantum Causal Structures). Il testo della conferenza è stato concepito e inizialmente redatto presso il Kavli Institute for Theoretical Physics, University of California, Santa Barbara, e supportato in parte dalla National Science Foundation con grant no. NSF PHY11-25915. I contenuti riguardanti la Causalità sono stati anche presentati a Londra presso la Royal Society l'11 dicembre 2017, alla conferenza Foundations of quantum mechanics and their impact on contemporary society, e poi pubblicati recentemente in Ref. [D'A18]. Le trasparenze di questa memoria sono disponibili sul sito web http://www.qubit.it.

\section{BIBLIOGRAFIA}

[ACP+16] Yakir Aharonov, Fabrizio Colombo, Sandu Popescu, Irene Sabadini, Daniele C. Struppa, and Jeff Tollaksen. Quantum violation of the pigeonhole principle and the nature of quantum correlations. Proceedings of the National Academy of Sciences, 113(3):532-535, 2016.

[Bar00] Julian Barbour. The end of time : the next revolution in physics. Oxford University Press, Oxford New York, 2000.

22 Come rimarcato in Ref. [DMP14], se la teoria non è causale esistono stati che non possono essere preparati con certezza. In altre parole, la procedura di postselezione non è generalmente disponibile. 
[BDP16] Alessandro Bisio, Giacomo Mauro D’Ariano, and Paolo Perinotti. Quantum cellular automaton theory of light. Annals of Physics, 368:177, 2016.

[Car16] Sean Carroll. The Big Picture: On the Origins of Life, Meaning, and the Universe Itself. Oneworld Publications, London, 2016.

[CDP11] Giulio Chiribella, Giacomo Mauro D'Ariano, and Paolo Perinotti. Informational derivation of quantum theory. Phys. Rev. A, 84(1):012311, 2011.

[Cox61] Richard T. Cox. The algebra of probable inference. Johns Hopkins University, Baltimore, 1961.

[D’A17] Giacomo Mauro D'Ariano. Dematerializzazione della fisica. In Realtà senza realismo, pages 11-34. Accademia di Scienze e Lettere dell'Istituto Lombardo, 2017.

[D'A18] Giacomo Mauro D'Ariano. Causality re-established. Philosophical Transactions of the Royal Society A, 376:20170313, 2018.

[Dav12] Paul Davies. That misterious flow. Scientific American, 21:8-13, 2012.

[DCP17] G. M. D'Ariano, G. Chiribella, and P. Perinotti. Quantum Theory from First Principles. Cambridge University Press, Cambridge, 2017.

[DMP14] Giacomo Mauro D'Ariano, Franco Manessi, and Paolo Perinotti. Determinism without causality. Physica Scripta, T163:014013, 2014.

[DP14] Giacomo Mauro D'Ariano and Paolo Perinotti. Derivation of the dirac equation from principles of information processing. Phys. Rev. A, 90:062106, Dec 2014.

[DT13] Giacomo Mauro D'Ariano and Alessandro Tosini. Emergence of spacetime from topologically homogeneous causal networks. Studies in History and Philosophy of Modern Physics, 2013.

[DY96] Giacomo Mauro D'Ariano and H. P. Yuen. Impossibility of measuring the wave function of a single quantum system. Phys. Rev. Lett., 76:2832-2835, Apr 1996.

[Gil00] Donald Gillies. Philosophical theories of probability. Psychology Press, 2000.

[Haa96] Rudolf Haag. Local quantum physics: Fields, particles, algebras, volume 2. Springer Berlin, 1996.

[Her82] N. Herbert. Flash-a superluminal communicator based upon a new kind of quantum measurement. Found. Phys., 12:1171, 1982.

[Jay03] Edwin T Jaynes. Probability theory: the logic of science. Cambridge University Press, Cambridge UK, 2003.

[Kai11] David Kaiser. How the hippies saved physics: Science, counterculture, and the quantum revival. WW Norton \& Company, 2011.

[Loc02] Robert Lockhart. Low-rank separable states are a set of measure zero within the set of low-rank states. Phys. Rev. A, 65:064304, Jun 2002.

[Pea12] Judea Pearl. Causality: Models, Reasoning, and Inference. Cambridge University Press, Cambridge, 2012.

[Pop59] Karl R. Popper. The Logic of Scientific Discovery. Routledge, London, 1959. [Rov17] Carlo Rovelli. L'ordine del tempo. Adelphi, Milano, 2017. 
[Rus12] Bertrand Russel. On the notion of cause. Proceedings of the Aristotelian society, 13:1-26, 1912.

[Shi83] Abner Shimony. In S. Kamefuchi et al., editor, Foundations of Quantum Mechanics in Light of the New Technology. Physics Society Tokyo, 1983.

[Shi86] Abner Shimony. In R. Penrose and C. Isham, editors, Quantum Concepts in Space and Time. Oxford, Claredon Press, 1986.

[vN96] John von Neumann. Mathematical foundations of quantum mechanics, volume 2. Princeton university press, Princeton, 1996.

[WZ82] William K Wootters and Wojciech H Zurek. A single quantum cannot be cloned. Nature, 299(5886):802-803, 1982. 